\title{
Decline in hospitalization risk and health care cost after initiation of depot antipsychotics in the treatment of schizophrenia
}

This article was published in the following Dove Press journal:

ClinicoEconomics and Outcomes Research

10 January 2011

Number of times this article has been viewed

\section{Xiaomei Peng \\ Haya Ascher-Svanum \\ Douglas Faries \\ Robert R Conley \\ Kory J Schuh}

Eli Lilly and Company, Indianapolis, IN, USA
Correspondence: Haya Ascher-Svanum Eli Lilly and Company, Lilly Corporate Center DC 4I33, Indianapolis, IN, 46285, USA

Tel +I 3 I7 27787 I3

Fax +I 3172767100

Email haya@lilly.com
Purpose: To assess change in hospitalization and cost of care from 6 months pre- to 6 months post-initiation on any depot antipsychotic among schizophrenia patients.

Patients and methods: Using a large United States commercial claims and encounters database, patients younger than 65 years diagnosed with schizophrenia were identified. Patients initiated on a depot antipsychotic were studied in a mirror-image design to assess change in hospitalization rates, mean duration hospitalized, and hospitalization cost. McNemar's test and paired $t$-tests compared the proportions of patients hospitalized and the mean duration. Paired $t$-test and bootstrapping methods compared costs.

Results: In these patients $(n=147)$, psychiatric hospitalizations declined from $49.7 \%$ pre-initiation to $22.4 \%$ post-initiation $(P<0.001)$, and the mean hospitalized duration for psychiatric purposes numerically declined from 7.3 to 4.7 days $(P=0.05)$. Total health care costs declined from $\$ 11,111$ to $\$ 7884(P<0.05)$ driven by reduction in costs for psychiatric hospitalizations from $\$ 5384$ to $\$ 2538(P<0.05)$.

Conclusion: Initiation of depot antipsychotic therapy appeared to be associated with a decline in hospitalization rates and costs. Current findings suggest that treatment with depot antipsychotics may be a cost-effective option for a subgroup of patients with schizophrenia who are at high risk of nonadherence with their oral antipsychotic medication regimen.

Keywords: mirror-image, claims database, treatment outcomes, depot antipsychotics

\section{Introduction}

Longer treatment duration with antipsychotics is associated with better clinical and functional outcomes. ${ }^{1,2}$ Despite the benefits, many patients have difficulty sustaining maintenance treatment because of difficulties adhering to daily regimens of oral medications. More than $35 \%$ of patients have adherence issues during their first 4 to 6 weeks of treatment ${ }^{3}$ and by 2 years, $75 \%$ are considered only partially adherent. ${ }^{4}$ A 1998 study reported that patients receiving antipsychotics took an average of only $58 \%$ of the recommended amount of the medications. ${ }^{5}$

Antipsychotics in long-acting injection form ("depot") were developed in the 1960 s to improve long-term schizophrenia treatment. ${ }^{6}$ Depot antipsychotics are often used to treat schizophrenia patients who are at high risk of nonadherence with their oral antipsychotics and, thus, also at a possible high risk of relapse and hospitalization. ${ }^{7,8}$ Treatment with a depot antipsychotic requires the patient to visit the clinic every 1 to 6 weeks to receive an intramuscular injection, which eliminates the patient's need to take the oral antipsychotic medication daily.

Although the efficacy of oral antipsychotic medications has been compared with depot medications by using randomized clinical trials (RCTs), there are difficulties 
using RCTs in this patient group. For example, the patients who are most appropriate for depot treatment tend to have additional problems such as substance abuse and legal issues, ${ }^{9}$ making them less likely to enroll in RCTs. Patients who are switched to depot antipsychotics generally have a history of poor adherence to oral antipsychotics and are frequently coaxed into depot treatment via legal commitments ("compulsory treatment") and thus unable to give a valid informed consent for a clinical trial. It has also been suggested that RCTs are likely to recruit adherent patients selectively by excluding patients with characteristics that are associated with poor adherence (eg, comorbid substance abuse). ${ }^{10}$ Yet these patients with poor adherence are the best candidates for depot treatment.

In contrast with RCTs, retrospective mirror-image studies do not require the patients to enroll in a study, as their medication and use of services in usual care are routinely captured in claims databases. Importantly, the mirror-image study design does not require a parallel active control group, as each patient serves as his or her own control. In these studies, patients maintained on oral medication are switched to depot medications and the outcome before and after the switch is compared. While some researchers reported a decline in the number of hospital admissions after initiation on depot antipsychotics, ${ }^{11,12}$ other researchers reported an increase in hospitalization days and resource utilization. ${ }^{13-15}$ Prior mirrorimage research publications have been confined to patients treated in the United Kingdom with one exception: one United States-based publication ${ }^{16}$ reported change in hospitalization and resource utilization after initiation of depot antipsychotics. In that study, from the Ohio Veterans Affairs (VA) Healthcare System, $75 \%$ of patients experienced a psychiatric-related hospitalization before depot initiation but only $42 \%$ were hospitalized during an equal amount of time after initiation. In addition to fewer psychiatric-related hospitalizations, these investigators reported shorter length of stay, fewer inpatient days per month, and one additional outpatient visit per month post-initiation. These researchers expressed a need for further United States studies in a non-VA population.

To fill this information gap and expand on the sparse United States-based research findings, the present mirror-image study aimed to assess change in hospitalization risk from 6 months pre- to 6 months post-initiation on any depot antipsychotic among patients treated for schizophrenia in the United States. Hospitalization risk was defined as the proportion of patients hospitalized and the number of psychiatric hospital admissions. Secondary objectives included assessment of the change in patients' adherence level, assessment of the change in utilization of outpatient services (emergency room, day treatment, and other outpatient visits), and assessment of the change in total direct cost and cost components (any inpatient hospitalization, psychiatric hospitalization, outpatient visits, and medication cost).

\section{Material and methods Data source}

The data source for this study was the Thomson Medstat MarketScan commercial claims and encounters databases (January 1, 2004 to March 31, 2008; MarketScan ${ }^{\circledR}$ Databases, Thomson Healthcare, Inc., Ann Arbor, MI). The databases capture person-specific clinical utilization, expenditures, and enrollment across inpatient, outpatient, prescription drug, and carve-out services from a selection of about 100 payers, including large employers, health plans, and government and public organizations. The MarketScan Databases link paid claims and encounter data over time and to detailed patient information across sites and types of providers, and over time. ${ }^{17}$

\section{Study sample selection}

The sample selection consisted of patients $(<65$ years of age) who were diagnosed with schizophrenia (International Classification of Diseases [ICD]-9-CM codes 295.XX) between January 1, 2004 and March 31, 2008 and who had at least 2 outpatient visits or 1 inpatient hospitalization associated with the schizophrenia diagnosis. Patients diagnosed with dementia type disorder were excluded. Patients who were initiated on any depot antipsychotic, who had no depot injection in the 6 months before this injection, and who had continuous enrollment for the 6 months before and 6 months after the depot initiation date ("index date") were included if the 2 outpatient visits or 1 inpatient hospitalization occurred within 180 days before the depot initiation. The index date was the date of the first depot injection.

\section{Study measures}

Patient demographics, including age and gender, were assessed for all patients. The specific antipsychotic depot medication, schizophrenia patients' related medical comorbidities, and substance use were determined based on ICD-9-CM diagnosis codes during the 6-month pre-index period. During the 6-month pre-index period and 6-month post-index period, data were collected on the proportion of patients hospitalized at least once for any reason, hospitalized at least once with a psychiatric diagnosis, and hospitalized at least once with a schizophrenia diagnosis, the number 
of psychiatric hospital admissions, total number of days hospitalized for psychiatric purposes, adherence with antipsychotic medication (defined as the Medication Possession Ratio [MPR] - the proportion of days the patient is in possession of any antipsychotic during each 180-day observation period), outpatient service use (emergency room, day treatment, and other office visits), total direct cost, and cost components (any hospitalization, psychiatric hospitalizations, outpatient services, and medication).

\section{Statistical methods}

Patients' baseline characteristics, comorbidities, and drug use disorders in the 6-month pre-index period were summarized. Analyses comparing the pre- vs post-initiation data employed McNemar's test for categorical variables, including the proportion of patients hospitalized for psychiatric reasons and the proportion of patients who used outpatient services, and paired $t$-tests to assess the continuous variables including the mean number of admissions, mean of total hospitalized duration, and MPR. Mean cost comparisons were conducted with paired $t$-tests and bootstrapping methods. Because cost data frequently do not exemplify a typical random distribution (ie, they are usually right-skewed and truncated at zero due to a small number of patients with high costs, a large number of patients with no costs, and the impossibility of costs less than zero $^{18}$ ), bootstrapping methods were used. Nonparametric bootstrapping is a technique where an empirical distribution of the mean cost difference between groups is constructed through resampling with replacement from the observed cost data. Bootstrapping is an alternative for analysis of cost data because it uses a nonparametric approach, which can directly address arithmetic means without making assumptions about the shape of the distribution. ${ }^{19}$ Bootstrap resampling (5000 iterations) was used to provide a nonparametric comparison of total cost as well as component costs (eg, any hospitalization cost, psychiatric hospitalization cost, outpatient cost, and medication cost) in the 6-month pre- vs post-index periods. No statistical adjustments were made for the multiple comparisons. Sensitivity analyses include examining the impact of acute care costs occurring just after the medication change, which may be incurred due to the failure on the prior treatment. ${ }^{20}$

\section{Results}

From a total of 674 patients with schizophrenia who were initiated on depot antipsychotics, data from 147 patients met inclusion criteria and were included in the analyses (Figure 1). Their baseline characteristics are presented in

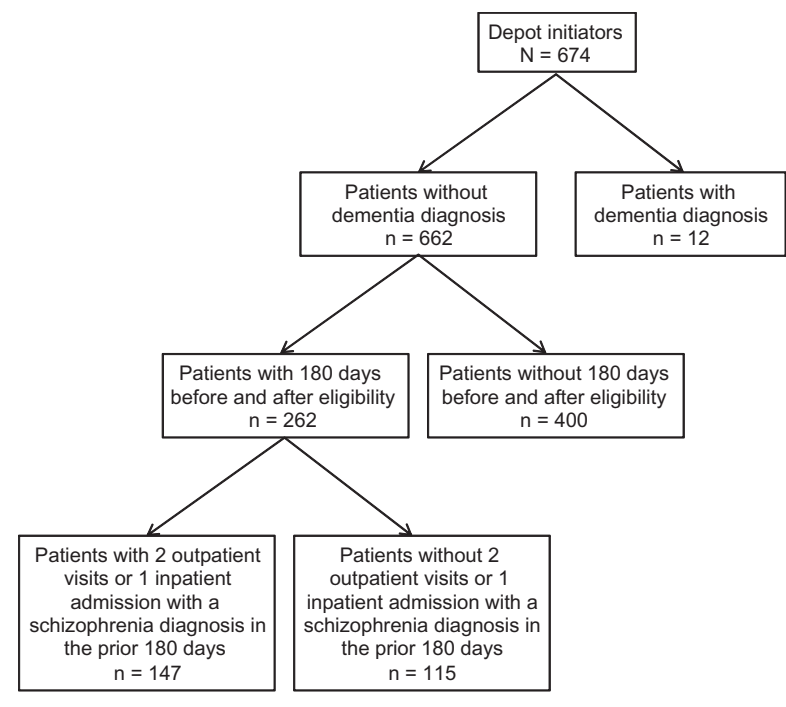

Figure I Patient flowchart.

Table 1 , showing that the mean age was the early 40 s and slightly more than half of patients were male.

After initiation of depot antipsychotics, patients improved their medication adherence. Mean antipsychotic MPR increased from $36.8 \%$ in the 6 months preceding depot initiation to $60.0 \%$ in the 6 months after initiation $(P<0.001)$. After depot initiation, patients were less likely to be hospitalized for any reason, for any psychiatric reason, and for schizophrenia specifically (Table 2). During the 6 months preceding initiation, 79 patients $(53.7 \%)$ were hospitalized for any reason compared with 44 patients $(29.9 \%)$ in the 6 months after initiation $(P<0.001)$. Hospitalization for any psychiatric reason decreased from 73 patients $(49.7 \%)$ to 33 patients $(22.4 \%$; $P<0.001)$. Hospitalization for schizophrenia decreased from 63 patients $(42.9 \%)$ to 30 patients $(20.4 \% ; P<0.001)$.

Table I Patient baseline characteristics

\begin{tabular}{|c|c|}
\hline No. of patients who initiated depot, $n$ & 147 \\
\hline \multicolumn{2}{|l|}{ Specific depot medication, n (\%) } \\
\hline Risperidone long-acting & $38(25.9)$ \\
\hline Haloperidol decanoate & $69(46.9)$ \\
\hline Fluphenazine decanoate & $40(27.2)$ \\
\hline Age at initiation, mean (SD) & $42.6(14.7)$ \\
\hline Male, n (\%) & $79(53.7)$ \\
\hline \multicolumn{2}{|l|}{ Medical comorbidities, n (\%) } \\
\hline Congestive heart failure & $3(2.0)$ \\
\hline Chronic pulmonary disease & $13(8.8)$ \\
\hline Mild liver disease & $4(2.7)$ \\
\hline Diabetes & $20(13.6)$ \\
\hline Diabetes with chronic complications & I (0.7) \\
\hline Substance use disorder, n (\%) & $17(1 \mid .6)$ \\
\hline Alcohol use, n (\%) & $4(2.7)$ \\
\hline Drug use, n (\%) & $14(9.5)$ \\
\hline
\end{tabular}

Abbreviation: SD, standard deviation. 
Table 2 Change in hospitalization parameters in the 6 months pre- vs 6 months post-depot initiation

\begin{tabular}{|c|c|c|c|}
\hline & $\begin{array}{l}\text { Before } \\
\text { initiation }\end{array}$ & $\begin{array}{l}\text { After } \\
\text { initiation }\end{array}$ & $P$-value ${ }^{a}$ \\
\hline $\begin{array}{l}\text { Proportion of patients } \\
\text { hospitalized at least } \\
\text { once for any reason, n (\%) }\end{array}$ & 79 (53.7) & 44 (29.9) & $<0.001$ \\
\hline $\begin{array}{l}\text { Proportion of patients } \\
\text { hospitalized at least once for } \\
\text { psychiatric reasons, n (\%) }\end{array}$ & $73(49.7)$ & $33(22.4)$ & $<0.00 \mathrm{I}$ \\
\hline $\begin{array}{l}\text { Proportion of patients } \\
\text { hospitalized at least once } \\
\text { for schizophrenia, n (\%) }\end{array}$ & $63(42.9)$ & $30(20.4)$ & $<0.001$ \\
\hline $\begin{array}{l}\text { Inpatient psychiatric } \\
\text { admissions, mean (SD) }\end{array}$ & $0.67(0.80)$ & $0.31(0.65)$ & $<0.001$ \\
\hline $\begin{array}{l}\text { Days hospitalized for any } \\
\text { reason, mean (SD) }\end{array}$ & $8.0(12.2)$ & $5.3(13.2)$ & 0.067 \\
\hline $\begin{array}{l}\text { Days hospitalized for } \\
\text { psychiatric reasons, mean (SD) }\end{array}$ & 7.27 (II.6) & $4.73(13.1)$ & 0.054 \\
\hline $\begin{array}{l}\text { Days hospitalized for } \\
\text { schizophrenia, mean (SD) }\end{array}$ & $5.7(9.2)$ & 4.0 (II.7) & 0.190 \\
\hline
\end{tabular}

The mean number of hospitalizations for any reason decreased from 0.78 to $0.41(P<0.001)$. The mean number of inpatient psychiatric admissions decreased from 0.67 to $0.31(P<0.001)$. The mean number of hospitalizations for schizophrenia decreased from 0.53 to $0.29(P=0.002)$. There were nonsignificant trends for decreases in the mean number of days of hospitalization for any reason (from 8.0 to 5.3; $P=0.067$ ) and for psychiatric hospitalization (from 7.3 to $4.7 ; P=0.054)$. The mean number of days of hospitalization for schizophrenia decreased from 5.7 to 4.0 but this change was not significant $(P=0.190)$.

During the 6 months preceding initiation, $36.1 \%$ of patients had 1 hospitalization for any reason, $12.2 \%$ had 2 , and $5.5 \%$ had 3 or more. In the 6 months after initiation, $21.8 \%$ had 1 hospitalization for any reason, $5.4 \%$ had 2 , and $2.7 \%$ had 3 or more. During the 6 months preceding initiation, $35.4 \%$ of patients had 1 hospitalization for psychiatric reasons, $12.2 \%$ had 2 , and $2.1 \%$ had 3 or more. In the 6 months after initiation, $16.3 \%$ had 1 hospitalization for psychiatric reasons, $4.1 \%$ had 2, and $2.0 \%$ had 3 or more. During the 6 months preceding initiation, $34.0 \%$ of patients had 1 hospitalization for schizophrenia, $7.5 \%$ had 2 , and $1.4 \%$ had 3 or more. In the 6 months after initiation, $14.3 \%$ had 1 hospitalization for schizophrenia, $4.1 \%$ had 2 , and $2.0 \%$ had 3 or more.

Change in outpatient service use was not significant. From the 6 months before depot initiation to the 6 months after initiation, the percent of patients who used emergency room services changed from $25.2 \%$ to $19.1 \%$, the percentage of patients who used day treatment changed from $56.5 \%$ to $53.7 \%$, and the percentage of patients having office visits changed from $88.4 \%$ to $86.4 \%$.

Mean total direct costs of treatment decreased from $\$ 11,111.30$ in the 6 months before depot initiation to $\$ 7883.80$ in the 6 months after initiation $(P<0.05$; Table 3). Median total direct costs decreased from $\$ 7089.40$ to $\$ 4051.93$. Mean total inpatient costs decreased from $\$ 6696.40$ to $\$ 3593.20(P<0.05)$ and psychiatric-related inpatient costs decreased from $\$ 5384.20$ to $\$ 2537.70$ $(P<0.05)$. Total outpatient costs and total medication costs did not change significantly. Median total outpatient costs decreased from $\$ 1591.05$ to $\$ 1297.62$. Median total medication costs were slightly changed $(\$ 853.04$ before depot initiation and $\$ 851.46$ after initiation). The sensitivity analyses indicated that mean total direct costs of treatment decreased from $\$ 10,615.60$ in the 6 months before depot initiation to $\$ 8379.50$ in the 6 months after initiation $(P<0.05)$.

\section{Discussion}

This study used a mirror-image design to assess and compare hospitalization risk and health care costs during the 6 months before and 6 months after initiation of depot antipsychotics for the treatment of patients with schizophrenia in the United States. This study found an improvement in medication adherence, a decrease in the rate of psychiatric hospitalization for any reason, for any psychiatric reason, and for schizophrenia specifically, and a decrease in health care costs after patients initiated depot antipsychotics. These results suggest that

Table 3 Change in total cost (US\$) and cost component in the 6 months pre- vs 6 months post-depot initiation

\begin{tabular}{llll}
\hline Mean (SD) & Six months pre-depot initiation & Six months post-depot initiation & P-value \\
\hline Total cost & $\$ 1 I, I I I .30(\$ 12,55 I .70)$ & $\$ 7883.80(\$ 948 I .30)$ & 0.003 \\
Total inpatient cost & $\$ 6696.40(\$ 10,620.40)$ & $\$ 3593.20(\$ 7806.20)$ & 0.002 \\
Psychiatric inpatient cost & $\$ 5384.20(\$ 8703.40)$ & $\$ 2537.70(\$ 6678.70)$ & $<0.001$ \\
Schizophrenia inpatient cost & $\$ 4143.30(\$ 7568.00)$ & $\$ 2377.30(\$ 6561.50)$ & 0.017 \\
Total outpatient cost & $\$ 2862.16(\$ 3681.64)$ & $\$ 2557.77(\$ 2919.90)$ & 0.300 \\
Total medication cost & $\$ 1552.82(\$ 2047.3 I)$ & $\$ 1732.78(\$ 27 \mid 3.65)$ & 0.220 \\
\hline
\end{tabular}

Abbreviation: SD, standard deviation. 
depot antipsychotic therapy may be a cost-effective option for a subgroup of patients typically at high risk of nonadherence with their oral antipsychotic regimen. ${ }^{9}$

Current findings are consistent with prior research in and outside the United States. A study in a VA population in the United States by Fuller et a ${ }^{16}$ found decreases in psychiatricrelated hospitalization from $75 \%$ to $42 \%$ after initiation of depot antipsychotics. In addition to fewer psychiatric-related hospitalizations, these researchers reported shorter length of stay, fewer inpatient days/month, and one additional outpatient visit/month post-initiation. Our results are also consistent with two United Kingdom studies that reported a decline in the proportion of patients requiring hospital admissions after initiation of depot antipsychotics. ${ }^{11,12}$ The Taylor et al ${ }^{11}$ study reported a decline in hospital admission rate from $62 \%$ before to $22 \%$ after initiation of depot. Similarly, the study by Niaz and Haddad ${ }^{12}$ found a reduction in hospital admissions, compulsory admissions, and total inpatient days.

Our study expanded on prior research by demonstrating an increase in medication adherence after initiating depot antipsychotics. This is important because patients being treated for schizophrenia often have problems with adherence to medications, and stopping medication often has serious consequences. ${ }^{21}$ Increased adherence with depot antipsychotics has the additional benefit of allowing clinicians to differentiate compliance failure from efficacy failure which can reduce the use of rescue medications and the need for switching to a secondchoice antipsychotic. ${ }^{22}$ Our study also demonstrated potential cost savings following depot initiation. Cost analyses found a significant decline in total cost of treatment, driven by decline in hospitalization cost from pre- to-post-depot initiation.

Results need to be considered in the context of the study limitations. The sample size was rather small $(n=147)$. Also, the study design is devoid of a control group. In this mirrorimage study, each patient served as his or her own control. As such, observed changes from pre- to post-depot initiation may reflect regression to the mean. Thus, we cannot determine if similar or even better results would have occurred with a different intervention. In addition, we used data from a large United States commercial claims and encounters database. Findings may not be generalizable to patients with schizophrenia who lack commercial insurance, which is a large segment of the schizophrenia population in the United States.

\section{Conclusion}

In summary, results from this study suggest that initiating depot antipsychotic therapy is associated with declines in hospitalization rates and related costs, compared with the prior treatment periods. These findings also suggest that treatment with depot antipsychotics may be a cost-effective option for a subgroup of patients with schizophrenia who are at high risk of nonadherence with their oral antipsychotic medication regimen.

\section{Acknowledgments/disclosure}

This work was supported by Eli Lilly and Company. All authors are full-time employees and minor shareholders of Eli Lilly and Company.

\section{References}

1. Liu-Seifert H, Adams DH, Kinon BJ. Discontinuation of treatment of schizophrenic patients is driven by poor symptom response: a pooled post-hoc analysis of four atypical antipsychotic drugs. BMC Med. 2005;3:21.

2. Dunayevich E, Ascher-Svanum H, Zhao F, et al. Longer time to antipsychotic treatment discontinuation for any cause is associated with better functional outcomes for patients with schizophrenia, schizophreniform disorder, or schizoaffective disorder. J Clin Psychiatry. 2007;68:1163-1171.

3. Chue P. Long-acting risperidone injection: efficacy, safety, and cost-effectiveness of the first long-acting atypical antipsychotic. Neuropsychiatr Dis Treat. 2007;3:13-39.

4. Weiden P, Zygmunt A. Medication noncompliance in schizophrenia. Part I. Assessment. J Pract Psychiatry Behav Health. 1997;3:106-110.

5. Cramer JA, Rosenheck R. Compliance with medication regimens for mental and physical disorders. Psychiatr Serv. 1998;49:196-201.

6. Simpson GM. A brief history of depot neuroleptics. J Clin Psychiatry. 1998;45:3-4.

7. Davis JM, Matalon L, Watanabe MD, Blake L. Depot antipsychotic drugs. Place in therapy. Drugs. 1994;47:741-773.

8. Kane JM, Aguglia E, Altamura AC, et al. Guidelines for depot antipsychotic treatment in schizophrenia. Eur Neuropsychopharmacol. 1998;8:55-66.

9. Shi L, Ascher-Svanum H, Zhu B, Faries D, Montgomery W, Marder SR. Characteristics and use patterns of patients taking first-generation depot antipsychotics or oral antipsychotics for schizophrenia. Psychiatr Serv. 2007;58:482-488.

10. Haddad PM, Taylor M, Niaz OS. First-generation antipsychotic longacting injections vs oral antipsychotics in schizophrenia: systematic review of randomised controlled trials and observational studies. Br J Psychiatry Suppl. 2009;52:S20-S28.

11. Taylor M, Currie A, Lloyd K, Price M, Peperell K. Impact of risperidone long acting injection on resource utilization in psychiatric secondary care. J Psychopharmacol. 2008;22:128-131.

12. Niaz OS, Haddad PM. Thirty-five months experience of risperidone long-acting injection in a UK psychiatric service including a mirrorimage analysis of in-patient care. Acta Psychiatr Scand. 2007;116: $36-46$.

13. Taylor DM, Young CL, Mace S, Patel MX. Early clinical experience with risperidone long-acting injection: a prospective, 6-month follow-up of 100 patients. J Clin Psychiatry. 2004;65:1076-1083.

14. Young CL, Taylor DM. Health resource utilization associated with switching to risperidone long-acting injection. Acta Psychiatr Scand. 2006;114:14-20.

15. Taylor D, Fischetti C, Sparshatt A, Thomas A, Bishara D, Cornelius V. Risperidone long-acting injection: a 6-year mirror-image study of healthcare resource use. Acta Psychiatr Scand. 2009;120:97-101.

16. Fuller M, Shermock K, Russo P, et al. Hospitalisation and resource utilisation in patients with schizophrenia following initiation of risperidone long-acting therapy in the Veterans Affairs Healthcare System. J Med Econ. 2009;12:317-324. 
17. Thomson Healthcare. MarketScan ${ }^{\circledR}$ research databases user guide and database dictionary. Ann Arbor, MI: Thomson Healthcare, Inc; 2007.

18. Ramsey S, Willke R, Briggs A, et al. Good research practices for costeffectiveness analysis alongside clinical trials: the ISPOR RCT-CEA Task Force report. Value Health. 2005;8:521-533.

19. Faries DE, Peng X, Obenchain RL. Costs and cost effectiveness analysis using propensity score bin bootstrapping. In: Faries DE, Leon AC, Haro JM, Obenchain RL, editors. Analysis of Observational Health Care Data Using SAS. Cary, NC: SAS Institute; 2010.
20. Faries DE, Nyhuis AW, Ascher-Svanum H. Methodological issues in assessing changes in costs pre- and post-medication switch: a schizophrenia study example. Cost Eff Resour Alloc. 2009;7:11.

21. Byerly MJ, Nakonezny PA, Lescouflair E. Antipsychotic medication adherence in schizophrenia. Psychiatr Clin North Am. 2007;30: 437-452.

22. Keith SJ, Kane JM. Partial compliance and patient consequences in schizophrenia: our patients can do better. J Clin Psychiatry. 2003;64: 1308-1315.

\section{Publish your work in this journal}

ClinicoEconomics \& Outcomes Research is an international, peerreviewed open-access journal focusing on Health Technology Assessment, Pharmacoeconomics and Outcomes Research in the areas of diagnosis, medical devices, and clinical, surgical and pharmacological intervention. The economic impact of health policy and health systems organization also constitute important areas of coverage. The manuscript management system is completely online and includes a very quick and fair peer-review system, which is all easy to use. Visit http://www.dovepress.com/testimonials.php to read real quotes from published authors.

Submit your manuscript here: http://www.dovepress.com/clinicoeconomics-and-outcomes-research-journal 